OBJECTIVE: Oral lichen planus (OLP) is chronic inflammatory disease of the oral mucosa, presenting in various clinical forms. The etiology of OLP is still unknown but mounting evidence points to the immunologic basis of this disorder.

Aim: Our study was undertaken to quantify the salivary levels of pro-inflammatory tumor necrosis factor-alpha (TNF- $\alpha$ ) in the reticular and the erosive/ atrophic forms of OLP, compared with age-matched healthy control volunteers.

Subjects and methods: Whole saliva from 40 patients with active lesions of OLP, as well as from 20 healthy persons, was investigated for the presence of TNF- $\alpha$ by enzyme immunoassay.

Results: Salivary TNF- $\alpha$ levels were significantlly increased in patients with OLP in comparison with healthy subjects. The presence of TNF- $\alpha$ showed positive correlation to clinical forms of OLP, being significantly higher in the erosive/atrophic type than in the reticular type of disease.

Conclusion: Saliva provides an ideal medium for the detection of pro-inflammatory markers of the oral cavity. In patients with OLP, TNF- $\alpha$ levels in saliva are elevated, correlating with the severity of illness. Salivary TNF- $\alpha$ analysis may be a useful diagnostic tool and a potential prognostic marker in OLP.

Key words: Oral lichen planus, Salivary levels, Tumour necrosis factor- $\alpha$

\section{Salivary levels of tumor necrosis factor- $\alpha$ in oral lichen planus}

\author{
Sonja Pezelj-Ribaric ${ }^{1, C A}$, Ivana Brekalo Prso², \\ Maja Abram ${ }^{3}$, Irena Glazar ${ }^{1}$, Gordana Brumini ${ }^{4}$ and \\ Marica Simunovic-Soskic ${ }^{5}$
}

${ }^{1}$ Department of Oral Medicine, ${ }^{2}$ Department of Dental Pathology, ${ }^{3}$ Department of Microbiology,

${ }^{4}$ Department of Informatics and ${ }^{5}$ Department of Prosthetic Dentistry, Medical Faculty, University of Rijeka, Brace Branchetta 20, 51000 Rijeka, Croatia

\author{
${ }^{\mathrm{CA}}$ Corresponding author \\ Tel: +38551213255 \\ Fax: +38551214467 \\ E-mail: sonja.pezelj-ribaric@ri.htnet.hr
}

\section{Introduction}

Oral lichen planus (OLP) is a relatively common mucocutaneous disease of unknown etiology. ${ }^{1}$ It is thought to affect about $0.5-2 \%$ of the world population, more often females at a mean age of onset in the fourth decade, although younger adults and children may be affected too. The cause of OLP is still not known, but cell-mediated immune dysfunction is implicated in the complex ethiopathogenesis of this disease. The immunologic process results in vacuolar degeneration, lysis and, ultimately, liquefaction of the basal cells. The large amount of cytokines released by affected keratinocytes and the associated inflammatory elements plays a key role in the selective recruitment of the $\mathrm{T}$ lymphocytes. T-celldominated infiltrate in the subepithelial region, which characterizes OLP, induces further release of chemokines and cytokines belonging to either the Th1 or Th2 groups. ${ }^{2}$ Such a mixed cytokine profile has been demonstrated by Simark-Mattsson et al., who found elevated concentrations of interleukin (IL)-2, IL-6 and IL-10, as well as tumor necrosis factor (TNF) $-\alpha$ and transforming growth factor- $\beta$ within the subepithelial infiltrate in OLP patients. ${ }^{3}$

TNF- $\alpha$ is a multifunctional cytokine that plays a prominent role in immune and host defense responses to infection, ${ }^{4}$ stimulates angiogenesis, ${ }^{5}$ influences tissue remodeling, ${ }^{6}$ and takes part in the regulation of cell proliferation and differentiation. It has been identified as an important mediator of cancer development $^{7}$ and a powerful activator not only of apoptotic, but also anti-apoptotic signaling cascades. ${ }^{8}$ Data collected during the past decade have pointed to TNF- $\alpha$ as a key cytokine in cutaneous lichen planus, as well as in OLP. ${ }^{10}$ High serum levels of TNF- $\alpha$ were detected in all patients with OLP in comparison with its hardly detectable levels in control subjects. ${ }^{11}$ Simultaneously with the expression of other pro-inflammatory and anti-inflammatory cytokines mRNAs, all the OLP lesions have been shown to contain cells with mRNA for TNF- $\alpha .{ }^{12}$

In this report we present data characterizing the pattern of TNF- $\alpha$ production in whole saliva of patients with OLP. We wanted to evaluate whether the salivary concentations of TNF- $\alpha$ were changed and whether they correlate with different clinical forms of OLP.

\section{Subjects and methods}

\section{Patients}

Forty patients with active lesions of OLP (20 with clinical diagnosis of the reticular form, and 20 with 
the erosive/atrophic form of disease) were included in this study. The clinical examination was performed according to the standard clinical criteria. Lesions were described as reticular if mainly lacelike hyperkeratotic patterns were presented. Lesions were described as erosive/atrophic if they exhibited erythematous change with few or aggressive ulcers. Clinical diagnosis of OLP was verified by an incisional biopsy. Twenty age-matched healthy volunteers served as a control group.

\section{Saliva collection and cytokine assay}

After informent consent had been obtained and medical, denture and social histories collected, each individual expectorated $10 \mathrm{ml}$ of unstimulated whole saliva into a sterile centrifuge tube. The saliva was centrifuged for $2 \mathrm{~min}$ at $9880 \times g$ and the clarified supernatant was filtered through a $0.45 \mu \mathrm{m}$ low protein binding membrane, separated into $0.5 \mathrm{ml}$ aliquots and frozen at $-80^{\circ} \mathrm{C}$ until use. Concentrations of TNF- $\alpha$ were determined using the human TNF- $\alpha$ ELISA kit purchased from SIGMA Immuno Chemicals (St Louis, MO, USA). The assay was performed according to the manufacturer's instructions, and the results are expressed in $\mathrm{pg} / \mathrm{ml}$. The detection limit for TNF- $\alpha$ was $4.4 \mathrm{pg} / \mathrm{ml}$, respectively. The test was performed in duplicate and repeated three times. The protein content was the results expressed in $\mathrm{pg} / \mathrm{ml}$.

\section{Statistical analysis}

Data are presented as median values, interquartile range (IQR) and on a logarithmic scale. The results obtained were compared using the non-parametric Wilcox test. A statistically significant difference was defined at $p<0.05$.

\section{Results}

Biopsed OLP tissues were stained with hematoxylin and eosin (HE staining) to evaluate the characteristic histopathological features of OLP. In each case the clinical diagnosis was confirmed by a biopsy with a histologic appearance that included a band-like, mainly lymphocytic, infiltrate in the connective tissue adjacent to the epithelial basement membrane, liquifaction degeneration of the basement membrane, and destruction of basal keratinocytes. The levels of TNF- $\alpha$ were measured in the whole saliva of healthy volunteers, as well as in patients with OLP, reticular form or erosive/atrophic form, and are presented as the median (IQR) on a logarithmic scale in Fig. 1.

Sufficient quantities of TNF- $\alpha$ (1.39 $\mathrm{pg} / \mathrm{ml}$, $\mathrm{IQR}=1.27-1.64)$ were present in all saliva samples obtained from healthy subjects. Salivary levels of TNF- $\alpha$ were elevated in all patients presenting symptomatic OLP. The median values $(2.83 \mathrm{pg} / \mathrm{ml}$, $\mathrm{IQR}=2.27-2.98)$ of TNF- $\alpha$ in saliva of the patients with the reticular form, as well as the erosive/ atrophic form $(3.08 \mathrm{pg} / \mathrm{ml}, \mathrm{IQR}=3.01-3.19)$, of the disease were significantly $(p<0.0001)$ higher in comparison with the values obtained from control subjects. A statistically significant difference $(p<0.001)$ was also demonstrated between the two, reticular versus erosive/atrophic, types of OLP, being more pronounced in the erosive/atrophic form, correlating with the severity of this malady.

\section{Discussion}

OLP is a persisting inflammatory keratotic disorder of oral mucous membranes. It may affect any part of the oral cavity, but the buccal mucosa, gingiva and tongue are the predominant sites. The disease occurs in a number of different clinical forms. ${ }^{1}$ However, reticular and erosive/atrophic forms are the most

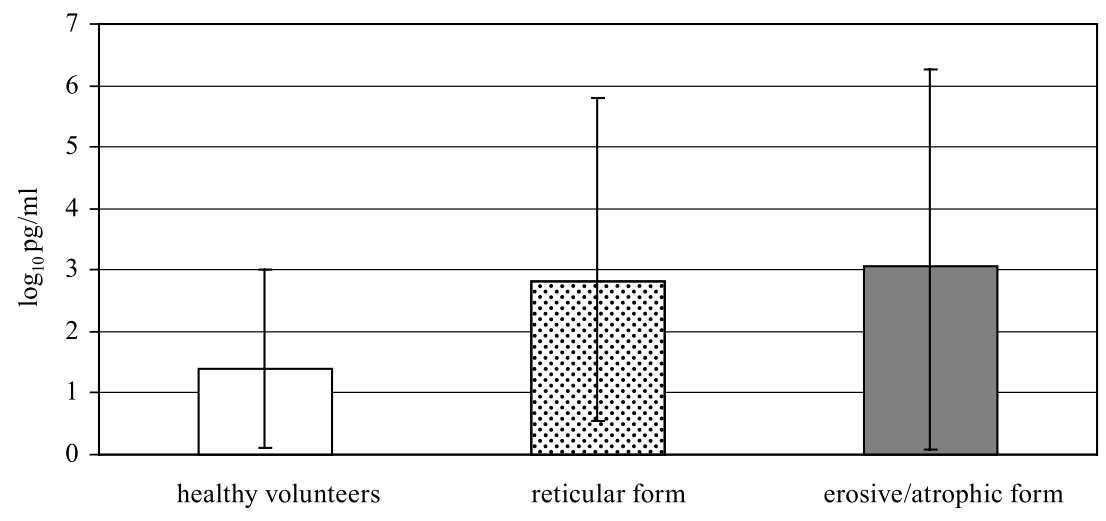

FIG. 1. The levels of TNF- $\alpha$ in the saliva samples from healthy volunteers and patients with the reticular form or the erosive/ atrophic form of OLP. Data represent the median $\log _{10}$ cytokine content (IQR) per milliliter of saliva derived from 20 subjects. Significance: healthy volunteers versus reticular and erosive/atrophic forms $(p<0.0001)$, and reticular form versus erosive/ atrophic form of OLP $(p<0.001)$. 
common clinical types of OLP. The etiology of this malady has not been fully elucidated, but it is generally accepted that immunological mecchanisms present the basis in the ethiopathogenesis of OLP. ${ }^{2}$ The immunologic process results in vacuolar degeneration of the germinative epithelial layer and destruction of the basal cells. Histochemical studies have identified $\mathrm{T}$ lymphocytes as a predominant infiltrating population. The major subset associated with the basal layer and responsible for the damage of keratinocytes is $\mathrm{CD}^{+}{ }^{+}$cytotoxic $\mathrm{T}$ cells. ${ }^{13} \mathrm{~T}$ lymphocytes that dominate the subepithelial region are major contributors of cytokines too. In addition, epidermal keratinocytes also appear to participate in producing cytokines in inflammatory lesions, perpetuating the condition by inducing adhesion molecule expression, playing a role in the selective recruitment of the T-cell-dominated infiltrate and inducing further cytokine release. $^{2}$ Despite that in OLP lesions mixed cytokines are simultaneously generated belonging to both Th1 and Th2 profiles, TNF- $\alpha$ has been implicated to have a key role in the initiation and progression of the disease. Elevated concentrations of TNF- $\alpha$ were detected in the sera ${ }^{11}$ and locally in the oral mucosal tissue of the OLP patients. Augmented mRNA levels detected in tissues by PCR seem to correlate with our results (i.e. with local protein synthesis). We found a significantly higher amount of TNF- $\alpha$ in all saliva samples obtained from patients with active OLP lesions in comparison with the healthy controls. Moreover, our data showed that salivary TNF- $\alpha$ concentrations varied in different clinical types of OLP, being particularly elevated in the case of the erosive/atrophic form of the disease. It is obvious that enhanced TNF- $\alpha$ production in saliva reflects clinical changes and correlates with the severity of OLP. A permanent or prolonged presence of high TNF- $\alpha$ amounts in saliva may contribute to malignant transformation of OLP lesions. It is known that TNF- $\alpha$ has a role in carcinogenesis, as well as that the erosive/atrophic form of OLP has a greater rate of malignant evolution in comparison with the reticular form of the disease, ${ }^{14}$ stressing the prognostic potential of TNF- $\alpha$ in saliva. As a diagnostic fluid, saliva is underused. It offers distinctive advantages over serum because it can be collected non-invasively by individuals with modest training. Furthermore, saliva analysis demonstrated comparable values of various biochemical and immunological parameters with those that are routinely evaluated in blood. ${ }^{15}$ Saliva has more diagnostic value that is generally appreciated and has promise as an aid in the diagnosis of systemic and esspecially oral pathologies.
OLP occupies an important place in dental practice because of the morbidity associated with oral lesions and their propensity for malignant development. Our data suggest an important role of high concentrations of TNF- $\alpha$ in perpetuation of the pathogenetical events in OLP. Inhibiting TNF- $\alpha$ activities in this disease may provide therapeutic benefits but more experimental and clinical studies are required for possible efficacy of drugs that can interfere with excess TNF- $\alpha$. Based on the presence of TNF- $\alpha$ in whole saliva we think that saliva analysis is a useful, worthwile tool in the diagnosis and follow-up of OLP, particularly in patients with erosive/atrophic affections of oral mucosa.

ACKNOWLEDGEMENT. This research was supported by a grant from the Ministry of Science and Technology (0062058), Republic of Croatia.

\section{References}

1. Mollaoglu N. Oral lichen planus: a review. Br J Oral Maxillofac Surg 2000; 38: $370-377$

2. Thornhill MH. Immune mechanisms in oral lichen planus. Acta Odontol Scand 2001; 59: 174-177.

3. Simark-Mattsson C, Bergenholtz G, Jontell M, et al. Distribution of interleukin-2, $-4,-10$, tumor necrosis factor- $\alpha$ and transforming growth factor- $\beta$ mRNAs in oral lichen planus. Arch Oral Biol 1999; 44: 499-507.

4. Abram M, Vuckovic D, Wraber B, Doric M. Plasma cytokine response in mice with bacterial infection. Mediat Inflamm 2000; 9: 229-234.

5. Nakao S, Kuwano T, Ishibashi T, Kuwano M, Ono M. Synergistic effect of TNF-alpha in soluble VCAM-1-induced angiogenesis through alpha 4 integrins. J Immunol 2003; 170: 5704-5711.

6. Gerstenfeld LC, Cho TJ, Kon T, et al. Impaired fracture healing in the absence of TNF-alpha signaling: the role of TNF-alpha in endochondral cartilage resorption. J Bone Miner Res 2003; 18: 1584-1592.

7. Scott KA, Moore RJ, Arnott CH, et al . An anti-tumor necrosis factor-alpha antibody inhibits the development of experimental skin tumors. $\mathrm{Mol}$ Cancer Ther 2003; 2: 445-451.

8. Victor FC, Gottlieb AB. TNF-alpha and apoptosis: implications for the pathogenesis and treatment of psoriasis. J Drugs Dermatol 2002; 1 $264-275$.

9. Simon M Jr, Gruschwitz MS. In situ expression and serum levels of tumour necrosis factor alpha receptors in patients with lichen planus. Acta Derm Venereol 1997; 77: 191-193.

10. Sugermann PB, Savage NW, Seymour GJ, Wals LJ. Is there a role for tumor necrosis factor-alpha (TNF-alpha) in oral lichen planus? J Oral Pathol Med 1996; 25: 219-224.

11. Yammamoto T, Yoneda K, Ueta E, Osaki T. Serum cytokines, interleukin2 receptor, and soluble intercellular adhesion molecule-1 in oral disorders. Oral Surg Oral Med Oral Pathol 1994; 78: 727-735.

12. Thornhil MH, Pemberton MN, Simmons RK, Theaker ED. Amalgamcontact hypersensitivity lesions and oral lichen planus. Oral Surg Oral Med Oral Pathol Oral Radiol Endod 2003; 95: 291-299.

13. Kawamura E, Nakamura S, Sasaki M, Ohyama Y, Kadena T, Kumamaru W, Shirasuna K. Accumulation of oligoclonal T cells in the infiltrating lymphocytes in oral lichen planus. J Oral Pathol Med 2003; 32: 282289

14. Rode M, Kogoj-Rode M. Malignant potential of teh reticular form of oral lichen planus over a 25 -year observation period in 55 patients from Slovenia. J Oral Sci 2002; 44: 109-111.

15. Nagler RM, Hershkovich O, Lischinsky S, Diamond E, Reznick AZ. Saliva analysis in the clinical setting: revisiting an underused diagnostic tool. $J$ Investig Med 2002; 50: 214-225.

\section{Received 16 December 2003 \\ Accepted 23 February 2004}




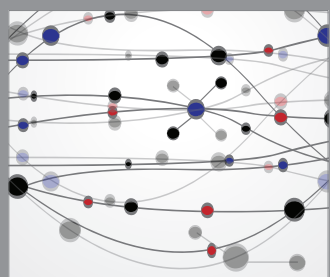

The Scientific World Journal
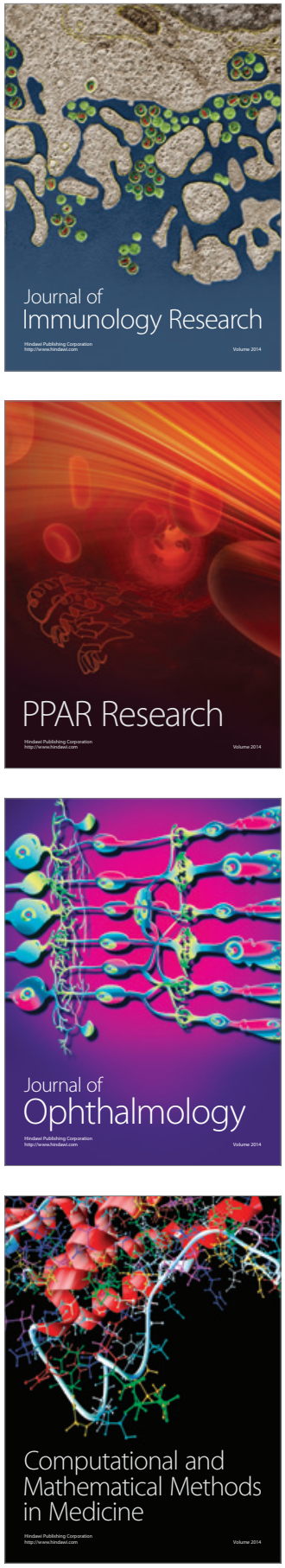

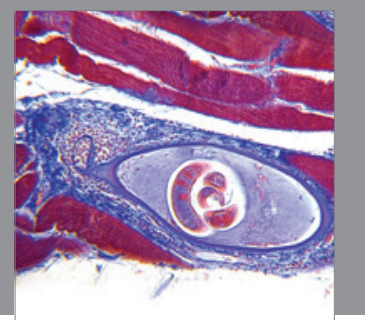

Gastroenterology

Research and Practice
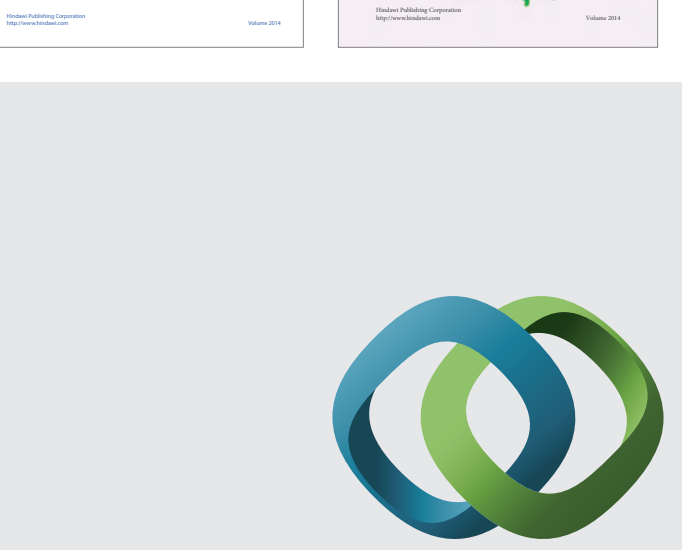

\section{Hindawi}

Submit your manuscripts at

http://www.hindawi.com
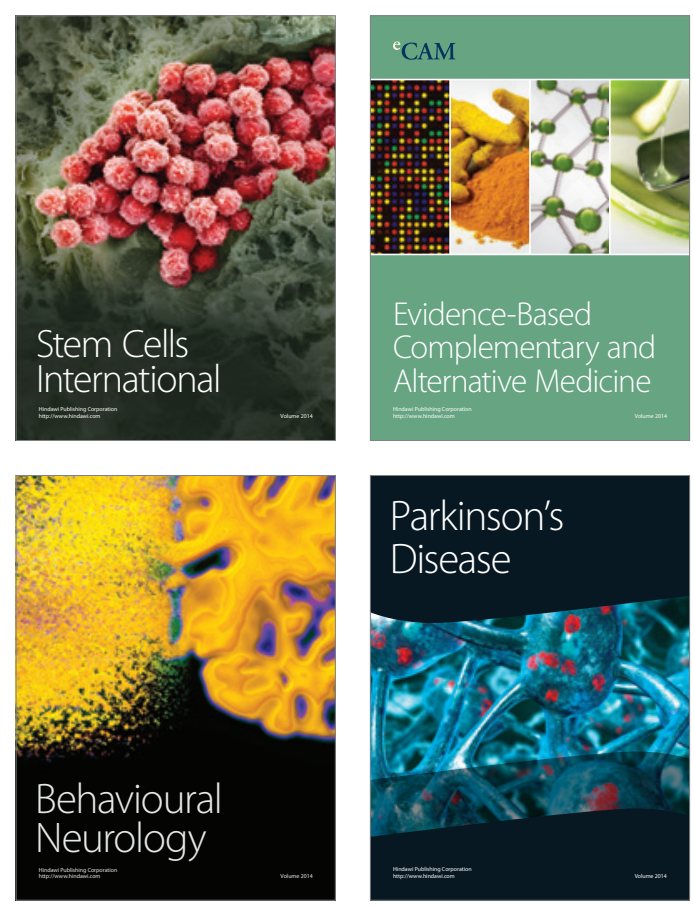

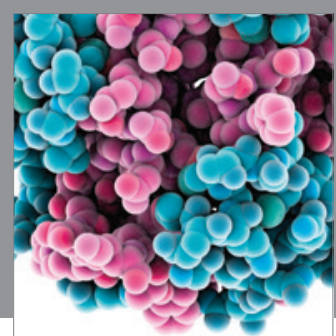

Journal of
Diabetes Research

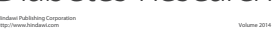

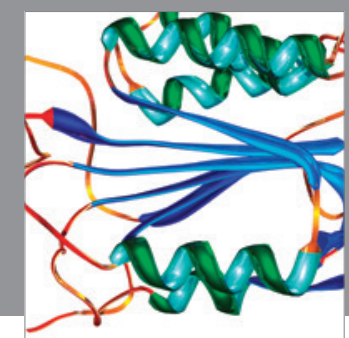

Disease Markers
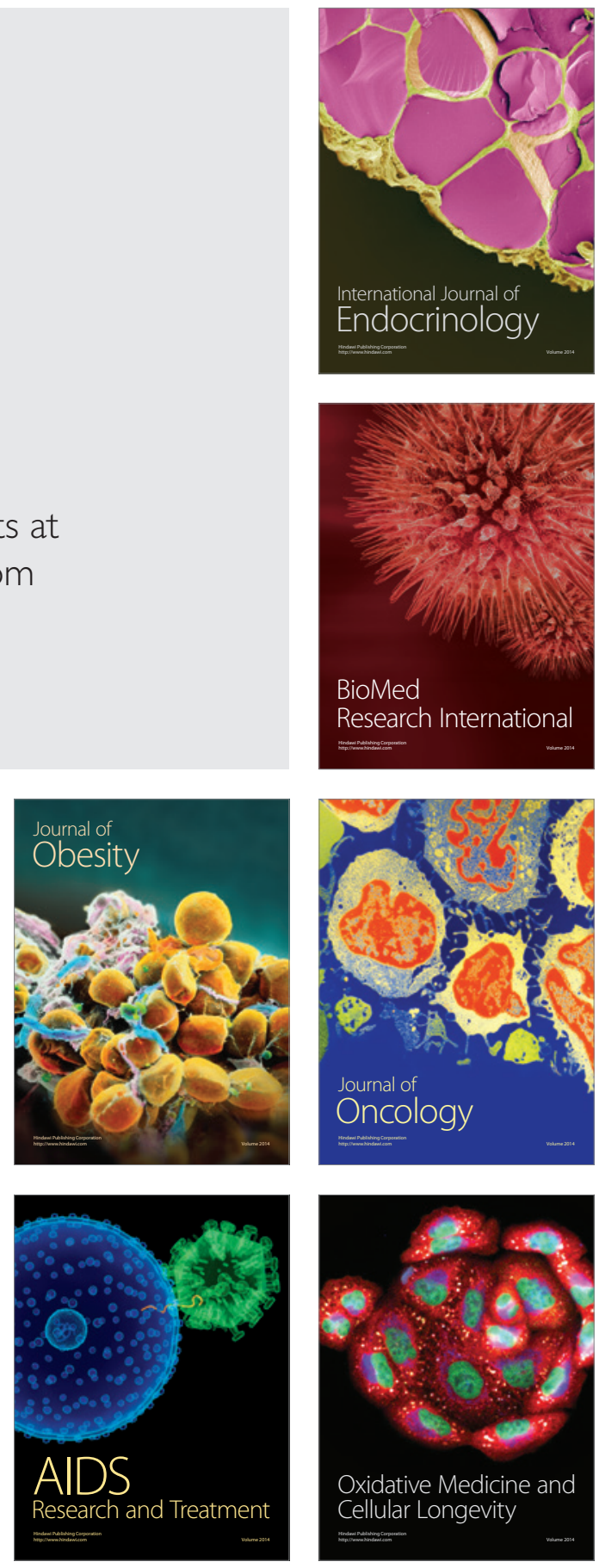